\title{
Perceptibility of Haptic Digital Watermarking of Virtual Textures
}

\author{
Domenico Prattichizzo ${ }^{\dagger}$ Mauro Barni ${ }^{\dagger}$ Hong Z. Tan ${ }^{*}$ Seungmoon Choi ${ }^{*}$ \\ (†) Department of Information Engineering, University of Siena, via Roma 56, 53100, Siena, Italy \\ (*) Haptic Interface Research Laboratory, Purdue University, West Lafayette, Indiana 47907 USA \\ E-mail:prattichizzo@ing.unisi.it,barni@ing.unisi.it,hongtan@purdue.edu,chois@purdue.edu
}

\begin{abstract}
Digital watermarking refers to the process of embedding a digital code into an image, video, printed document or audio media. The code should not interfere with the normal use of the media but can be recovered later. With the growing interest in haptic interactions for training and museum displays, it is only a matter of time that haptic digital media become available on the internet. For example, one will soon be able to feel the shape of Michaelangelo's David or Japan's Big Buddha by downloading a file that can be "played" on a force-feedback device. The haptic file may contain surface texture data in addition to topography so that David feels like marble and Big Buddha wood. In the meanwhile, the need for haptic digital watermarking will arise for protecting haptic media contents. This paper introduces for the first time the idea of haptic watermarking and shows one way to embed a watermark into a host surface texture signal. We also demonstrate the imperceptibility of the texture watermark in a psychophysical experiment.
\end{abstract}

\section{Introduction}

In the last decade, digital watermarking has received increasing attention as a tool for copyright protection of digital data $[1,2]$. According to the watermarking paradigm, data protection is achieved by injecting into the data an invisible signal, i.e. the watermark conveying information about ownership, data provenance or any other information that can be useful to enforce copyright laws. A great deal of research has focused on digital watermarking of audio, images and video. On the contrary, watermarking of 3D objects is far from this level of maturity although 3D models are widely used in several applications such as virtual prototyping, cultural heritage, and entertainment industry. One of the reasons for this gap is that it is difficult to extend common processing algorithms used in signal processing to $3 \mathrm{D}$ data.
The first requirement that any watermarking technique must satisfy is watermark imperceptibility. In the case of still images and video sequences, the imperceptibility requirement has triggered a great deal of research about the Human Visual System (HVS), resulting in a number of possible algorithms which exploit the properties of the HVS to improve watermark invisibility while keeping the watermark energy constant [3]. Something similar happened for the case of $3 \mathrm{D}$ watermarking, where the intrusiveness of the watermark is judged in term of its visibility in the rendered version of the mesh [4].

While recognizing the importance of these studies, though, it should not be forgotten that other modalities exist to sense a 3D mesh. This is certainly the case where the underlying 3D model is touched by means of haptic interfaces. We foresee in the not so distant future that lowend haptic interfaces may become commodity and therefore demand haptic files that can be downloaded from the internet to keep their users entertained. This will create a need for the protection of haptic contents and propel haptic watermarking technology.

Haptic interfaces allow physical interactions with virtual 3D objects through the sense of touch. Despite an enormous increase in research activity in the last few years, the science of haptics (perception and manipulation with our hands) is still a technology in its infancy [5]. State of the art consists of three degrees-of-freedom interfaces, i.e. devices with three motors that are able to actuate three linear forces at the end effector. The most well known and commercially successful interfaces of this kind are the PHANTOM, produced by SensAble Technologies (www.sensable.com), and the Delta, produced by ForceDimension (www.forcedimension.com). These are devices that interact with the virtual environment through one contact point only. Interfaces with higher number of DOFs and with multiple interaction points are also available, but are less common or reliable than those with three DOFs and one interaction point.

With the term haptic rendering we refer to a branch of haptics research that deals with calculating the right inter- 
action force between a virtual representation of the user and a virtual object. In most cases, haptic rendering is a two-step process consisting of shape and texture rendering. In this context, shape refers to the macrogeometry of an object surface, as supposed to texture that describe the fine structure, or microgeometry of the surface [6]. To render the shape of an object, we can use typical single point contact rendering algorithms such as god-object [7] and the virtual proxy [8]. To render the texture, we can perturb the force for the object shape based on a texture model (for example, see [9, 10]; see also [11] for a review of texture rendering techniques).

As to watermarking, it is evident that in order to fully guarantee the imperceptibility of the watermark, the possibility of sensing it through a haptic interface must be taken into account. It is the scope of this paper to introduce a simple watermarking technique for haptic data and present a psychophysical experiment that demonstrated the imperceptibility of a watermark signal superimposed onto a host signal playing the role of the to-be-protected data. More specifically, we investigated the perceptibility of haptic watermarks embedded in the textures of a virtual object. To simplify the problem, we considered a sinusoidal watermark superimposed to a sinusoidal host signal. Despite its simplicity, this experiment permits us to demonstrate that, at least in a simplified framework, it is possible to create a watermark which is haptically imperceptible and, at the same time, detectable by means of a spectral analysis.

This paper is organized as follows. Section 1 introduces the digital watermarking problem. In Section 2 a brief overview of digital watermarking is given. Section 3 presents the haptic watermarking idea. In Section 4, the psychophysical experiment we carried out is described. The main results of the experiment are discussed in Sections 5 and 6 . The paper ends with section 7 where some possible directions for future research are highlighted.

\section{Overview of watermarking techniques}

In this section we give a brief overview of digital watermarking. Generally speaking any watermarking system can be seen as a communication system consisting of two main parts: a watermark embedder, and a watermark detector. The watermark is transmitted through the watermark embedder over the original to-be-marked object (in our case a 3D surface). The watermark detector extracts the watermark from the marked data. Intentional and unintentional attacks and distortions applied to the mesh hosting the watermark further characterize and complicate the transmission channel. As to the watermark, it usually consists of a pseudo-random sequence with uniform, binary or Gaussian distribution.

According to the set of features the watermark is injected into, watermarking techniques can be divided into two main categories: (i) spatial/temporal domain techniques that directly add the watermark to pixel values; and (ii) transformed domain techniques that add the watermark in the frequency domain.

Once the host features have been chosen, the embedding rule has to be specified. The two most common approaches to watermark embedding are the additive and multiplicative methods. In the additive method, $y_{i}=x_{i}+\gamma w_{i}$, where $x_{i}$ is the $i$-th component of the original feature vector, $w_{i}$ the $i$-th sample of the watermark, $\gamma$ a parameter controlling the watermark strength, and $y_{i}$ the $i$-th component of the watermarked feature vector. In the multiplicative method, $y_{i}=x_{i}+\gamma w_{i} x_{i}$. Recently a new approach to watermark embedding has been proposed. This approach, commonly referred to as informed watermarking or QIM (Quantization Index Modulation) watermarking [12], can greatly improve the performance of the system as a whole. However, a discussion on this method is beyond the scope of this paper and will be a topic for future research.

After watermark insertion, a perceptual hiding step is sometimes performed to make the watermark less perceivable to the eye (or to other human sensory modalities).

Among the characteristics of watermarking algorithms, a crucial role is played by the way the watermark is extracted from data. In blind decoding, the decoder does not need the original data (mesh) or any information derived from it in order to recover the watermark. Conversely, nonblind decoding refers to a situation where extraction is accomplished with the aid of the original, non-marked data. In spite of the benefits the latter offers in terms of robustness, non-blind decoding is not desirable in many applications, where the availability of the original data can not be guaranteed. An important distinction can also be made between algorithms embedding a mark that can be read (i.e. the bits contained in the watermark can be read without knowing them in advance) and those inserting a code that can only be detected. In the former case, the bits contained in the watermark can be read without knowing them in advance. In the latter case, one can only verify if a given code is present in the document; i.e. the watermark can only be revealed if its content is known. It is common to refer to the extraction of a readable watermark with the term watermark decoding, whereas the term watermark detection is used for the extraction of a detectable watermark.

Watermark detection is a typical binary hypothesis testing problem. Given an observation variable, a decision rule is defined to decide whether the watermark is present (hypothesis $H_{1}$ ) or not (hypothesis $H_{0}$ ). For example, in correlation-based detection, the observation variable is the correlation $\rho$ between the watermark and the host features. To decide whether the watermark is present or not, $\rho$ is compared to a threshold $T_{\rho}$, which is usually set by minimizing the missed detection probability subject to a maximum false detection rate (Neyman-Pearson criterion [13]). Decoding of a readable watermark is slightly differ- 
ent. Without going into details, it suffices to say that the problem is one of receiving a signal transmitted through a very noisy channel.

Readers interested in a more detailed discussion of watermarking issues may refer to [14].

To test the idea of haptic watermarking, we conducted a psychophysical experiment aimed at evaluating the perceptibility of watermarks embedded in a virtual haptic texture.

\section{Digital watermarking of haptic texture}

As mentioned earlier, texture refers to the fine structure, or microgeometry, of an object's surface, as supposed to shape that describes a surface's macrogeometry [6]. Just like real textures can be explored by rubbing a fingerpad or a pencil-like stylus against it, virtual haptic textures can be displayed on a multi-pin array that allows direct finger contact, or on a force-feedback device that allows single-point contact mediated by a stylus. In the latter case which is more commonplace, texture information is conveyed through vibration $[15,16,17,18,19]$.

For sinusoidal texture models that are frequently used to render haptic textures, previous work has shown that the temporal signal contributing to texture perception is characterized by a spectral peak of the force or position signals recorded near or at the stylus tip [11]. The frequency of this peak is determined by the spatial period of the sinusoidal grating and the speed at which it is stroked. The amplitude of the peak determines the perceived intensity (or roughness) of the texture. It now follows that digital watermarking of virtual haptic texture can be considered in the spectral domain: Given a host texture signal, an additional spectral peak at a different frequency with an amplitude below human detection threshold (the minimum signal strength required for producing a sensation; such data are widely available in the literature for various experimental conditions; see [18] for example) can be added that guarantees its imperceptibility. Therefore, this initial experiment employed a simplified version of the additive method outlined in the previous section.

\section{Experiment methods}

\subsection{Subjects}

Five subjects, aged 25-39, participated in the experiment. All are right-handed with no known sensorimotor impairments with their hands. Their prior experience with the PHANToM device varied from naïve to expert. All subjects gave their written consent to the experimental protocol that was approved by the IRB at Purdue University.

\subsection{Stimuli}

We asked human subjects to explore textured flat virtual surfaces using a PHANToM force-feedback device (model 1.0A, SensAble Technologies, Inc., Woburn, MA, USA). The surface was modelled as a vertical plane in front of the subject, and the texture as a one-dimensional sinusoidal grating superimposed on the plane (Fig. 1). The height map of the host texture signal was defined by

$$
h(x)=A_{h} \sin \left(\frac{2 \pi}{L_{h}} x\right)+A_{h}
$$

where $A_{h}=1 \mathrm{~mm}$ and $L_{h}=2 \mathrm{~mm}$. The watermarked texture signal was defined by

$$
h(x)=A_{h} \sin \left(\frac{2 \pi}{L_{h}} x\right)+A_{h}+A_{w} \sin \left(\frac{2 \pi}{L_{w}} x\right)+A_{w}
$$

where $L_{w}=5 \mathrm{~mm}$, and $A_{w}$ was 0.2 or $0.5 \mathrm{~mm}$ for condition 1 or 2 , respectively. We hasten to point out that it is much more meaningful to test the perceptibility of watermarks embedded in a texture defined by a height map that can not be fully represented by an implicit function. The host and watermark texture signals used in this experiment can be viewed as one component of a more complicated height map.

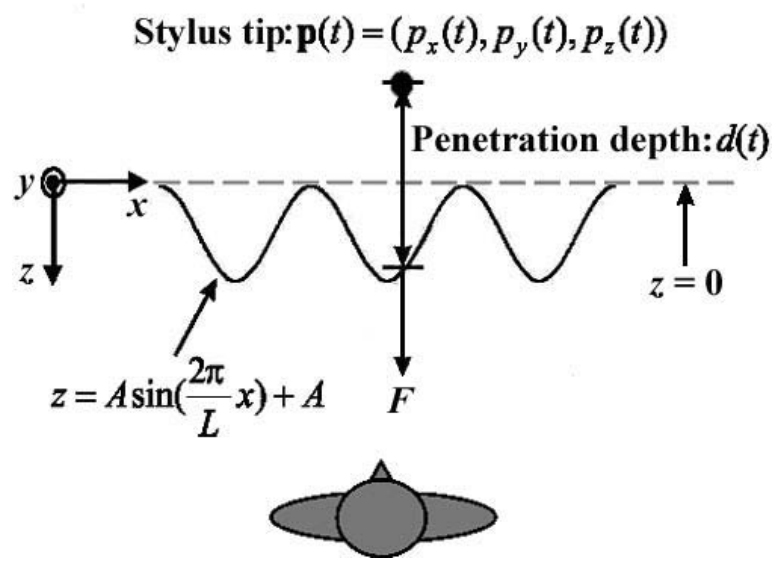

Figure 1: Bird's-eye view of subject, textured vertical plane, and coordinate frame. The dashed line indicate the flat vertical plane upon which a one-dimensional sinusoidal texture model is superimposed. Subjects stroked the textured surface along the $x$-axis. Penetration depth is measured as the distance between the stylus tip and the point on the textured surface along the $z$-axis.

Subjects held the stylus of the PHANToM with their right hand and stroked the textured surface back and forth along the $x$-axis. Whenever the stylus tip was inside the virtual texture, a restoring force ( $\boldsymbol{F}$ in Fig. 1) was applied 
to the subjects' hands. The force vector felt by the subjects was always along the $z$-direction with a magnitude of $K d(t)$, where $K=0.3 \mathrm{~N} / \mathrm{mm}$ was the stiffness of the virtual textured surface. The penetration depth $d(t)$ was calculated as $d(t)=h\left(p_{x}(t)\right)-p_{z}(t)$ whenever the stylus tip was inside the virtual surface. When the stylus was outside the virtual textured surface, no force was displayed. Subjects perceived the surface texture by experiencing force variations during lateral stroking.

\subsection{Conditions}

We examined the perceptibility of haptic watermarks by asking subjects to discriminate two virtual textures: one with a host signal alone, and the other with the host signal and an embedded watermark. The host signal was a onedimensional sinusoidal grating with an amplitude $A_{h}=1$ $\mathrm{mm}$ and a spatial period $L_{h}=2 \mathrm{~mm}$ (Fig. 2, top trace). Two watermark signals, also one-dimensional sinusoidal gratings, were chosen with $A_{w}=0.2$ (or $0.5 \mathrm{~mm}$ ) and $L_{w}=5$ $\mathrm{mm}$. The two amplitudes of the watermark signals were selected to be below or above human detection thresholds, respectively. In the spatial domain, the watermarked texture signal was a modulated sinusoidal signal (Fig. 2, bottom trace). In the frequency domain, it exhibited two spectral peaks (Fig. 3). The upper panel in Fig. 3 shows the spectral density of $p_{z}(t)$ (solid line) for condition 1 where the weaker watermark signal was embedded in the host texture signal. The $p_{z}(t)$ data were recorded from a single stroke of the watermarked textured surface using the PHANToM haptic device. The dashed line in the same panel shows the human detection thresholds taken from the literature [20]. The two spectral peaks corresponded to the watermark $(\approx$ $40 \mathrm{~Hz})$ and host $(\approx 76 \mathrm{~Hz})$ signals, respectively. The lower panel in Fig. 3 shows the same for condition 2 where the stronger watermark signal was used. The perceptibility of the two watermarks could be predicted by comparing the watermark peaks with the corresponding human detection thresholds. Since the peak for the weaker watermark was at roughly the same level as the human detection threshold, we did not expect the subjects to be able to detect it. The peak for the stronger watermark, however, was clearly above the human detection threshold. We therefore expected this watermark to be easily perceived by our subjects.

\subsection{Procedure}

A one-interval two-alternatives forced-choice paradigm was used. On each trial, the subject felt either the host texture alone, or the host texture with watermark. Their task was to respond " 1 " to the host texture and "2" to the watermarked host texture. No trial-by-trial correct-answer feedback was provided during data collection. Each subject performed four 100-trial blocked runs, two for con-

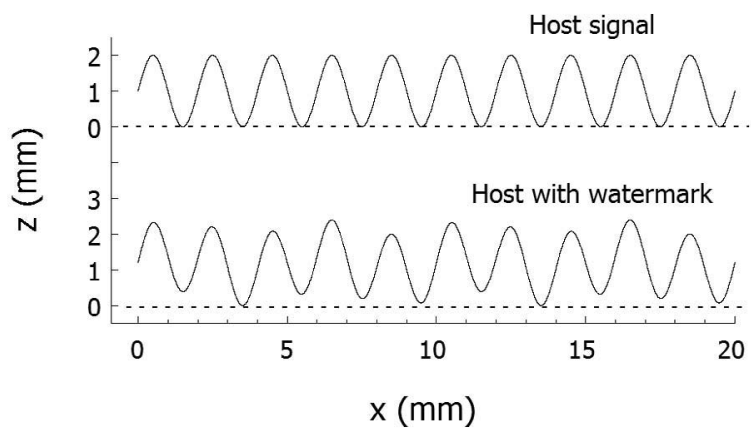

Figure 2: Spatial representation of stimuli. Top trace shows the $z$ vs. $x$ sinusoidal grating for the host texture alone $\left(A_{h}=1 \mathrm{~mm}, L_{h}=2 \mathrm{~mm}\right)$. Bottom trace shows the same host signal with an embedded watermark ( $A_{w}=0.2$ $\left.\mathrm{mm}, L_{w}=5 \mathrm{~mm}\right)$.

dition 1 and two for condition 2. The order of the four runs was randomized for each subject. At the beginning of each run, subjects familiarized themselves with the stimuli by entering either 1 or 2 on a keyboard to feel the corresponding texture. Training was terminated by the subjects whenever they were ready.

\section{Data analysis and results}

Data from each condition formed a $2 \times 2$ stimulusresponse matrix consisting of 200 trials. Instead of calculating the percent-correct scores which are often confounded by subjects' response biases, we estimated the sensitivity index $d^{\prime}$ that provided a bias-free measure of the discriminability between the host and watermarked host textures (i.e., the perceptibility of the watermark signal) [21]. In this method of data processing, it is assumed that the underlying density functions associated with the two stimuli being discriminated are normal and of equal variances (means $M_{1}$ and $M_{2}$, and variance $\sigma$ ). The sensitivity index $d^{\prime}$ is then defined as the normalized difference between the means: $d^{\prime}=\left(M_{1}-M_{2}\right) / \sigma$. A $d^{\prime}$ value of 0.0 , 1.0 or 2.0 corresponds to a percent-correct score of $50 \%$, $69 \%$ or $84 \%$, respectively, assuming no response biases. With 200 trials collected for each subject per experimental condition, we were also able to estimate the standard deviations of $d^{\prime}$ values [22].

Shown in Fig. 4 are the values of sensitivity index $d^{\prime}$ for five subjects. The $d^{\prime}$ values were essentially 0 in condition 1 where the weaker watermark signal was used, indicating that the subjects could not tell the difference between the host texture alone and the watermarked texture. In condition 2 where the stronger watermark signal was used, the values of $d^{\prime}$ were in the range $1.39-2.63$ indicating high 


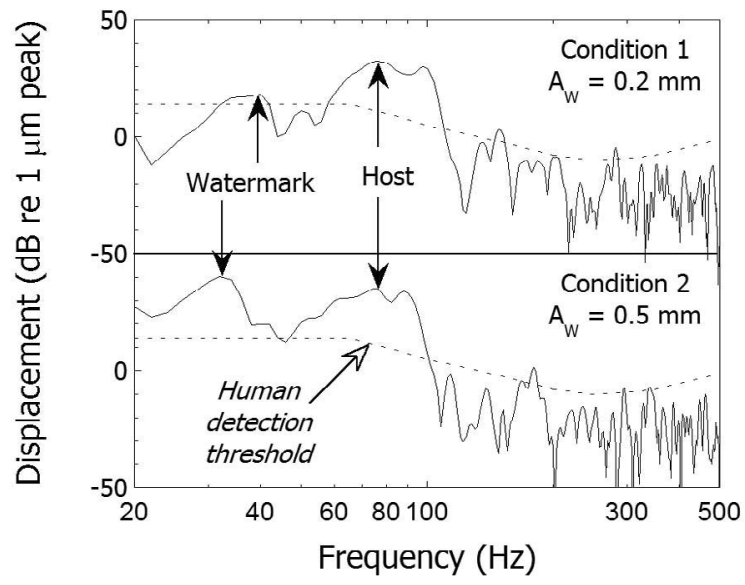

Figure 3: Power spectral densities of $p_{z}(t)$ for the two watermarked textures (solid lines) and human detection thresholds (dashed lines). The upper and bottom panels correspond to the weaker and stronger watermarks, respectively. The locations of the spectral peaks corresponding to the watermark and the host textures are indicated by arrows.

discriminability. Therefore, the stronger watermark signal was clearly perceivable to all the subjects. These results confirm our earlier predictions about the perceptibility of the two watermark signals.

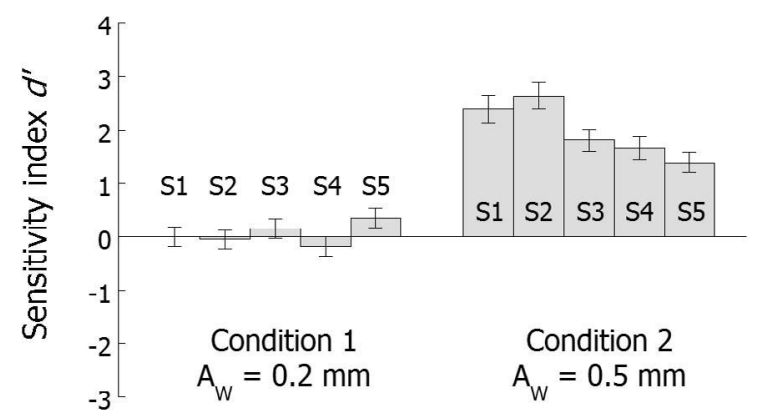

Figure 4: Experimental results. Shown are the sensitivity indices and the corresponding standard deviations for subjects S1-S5 under the two watermarking conditions.

\section{Discussion}

The spectral-domain analysis technique described here serves several purposes in the design and detection of haptic texture watermarks using the additive watermarking technique. First of all, the technique is useful for predicting the perceptibility of watermarks. Instead of asking many subjects to stroke several virtual textures for hundreds of times each and report whether a watermark is felt, a watermark designer only needs to stroke the watermarked texture once. The position signals during stroking can be readily recorded from the encoders in any force-feedback device. The perceptibility of the watermark can then be assessed by comparing the spectral peak of the watermark to the human detection threshold at the same frequency. This approach is much less time consuming than running psychophysical experiments for all conceivable watermark signals. Secondly, the technique is useful for detecting watermarks. The frequency of the host or the watermark texture signal is around $|v| / L$ where $v$ is the average stroking velocity and $L$ the spatial period of the sinusoidal grating [11]. The average stroking velocity can be estimated from the position data along the lateral stroking direction [ $p_{x}(t)$ in Fig. 1]. One can then look for a spectral peak near $|v| / L_{w}$ to detect the watermark. Thirdly, our approach can be easily extended to surface shapes other than a flat plane. It can also be extended to many other texture models, such as square-wave gratings, by decomposing the texture model into a Fourier series. We believe that this is the first time that anyone has performed a perceptibility analysis on haptic watermarking of virtual textures.

\section{Future work}

With this work we have taken a first step towards the analysis of the haptic perceptibility of digital watermarks. However, it goes without saying that a lot of work is still ahed of us. Our future work will concentrate on the study of the haptic perceptibility of more realistic watermarking signals, such as noise-like and QIM watermarks. We will also consider the watermarking of 3D meshes instead of virtual textures represented by explicit surfaces. Finally, we are planning to compare haptic and visual perceptibility, in order to analyze whether the constraints set by the haptic channel are more or less stringent than and follow the same rules of visual constraints.

\section{Acknowledgments}

This work was supported in part by Fondazione Monte dei Paschi di Siena and by Univerità di Siena under Grant PAR-2003, by National Science Foundation awards under Grant 0098443-IIS and 0328984-CCF, and by the Purdue University Envision Centre for Data Perceptualization.

\section{References}

[1] M. Barni and F. Bartolini, "Data hiding for fighting piracy," Signal Processing Magazine, vol. 21, no. 2, pp. 28-39, March 2004. 
[2] M. Maes, T. Kalker, J.-P Linnartz, J. Talstraand F. G. Depovere, and J. Haitsma, "Digital watermarking for DVD video copy protection," IEEE Signal Processing Magazine, vol. 17, no. 5, pp. 47-57, September 2000.

[3] R. B. Wolfgang, C. I. Podilchuk, and E. J. Delp, "Perceptual watermarks for digital images and video," Proceedings of the IEEE, vol. 87, no. 7, pp. 11081126, July 1999.

[4] M. Corsini, F. Uccheddu, F. Bartolini, M. Barni, R. Caldelli, and V. Cappellini, "3D watermarking technology: Visual quality aspects," in Proc. 9th Conf. Virtual System and Multimedia, VSMM'03, Quebec - Canada, October 2003.

[5] J.K. Salisbury, "Making graphics physically tangible," Communications of the ACM, vol. 42, no. n, Aug. 1999.

[6] D. Katz, The World of Touch, Lawrence Erlbaum Associates, Hillsdale, NJ, 1925/1989.

[7] C. Zilles and K. Salisbury, "A constraint-based godobject method for haptic display," in Proc. IEEE/RSJ Int. Symp. Intelligent Robots and Systems, 1995, vol. 3 .

[8] D. Ruspini, K. Kolarov, , and O. Khatib, "The haptic display of complex graphical environments," in $A n$ nual Conference Series on Computer Graphics, 1997, pp. 345-352.

[9] T.H. Massie, "Initial haptic explorations with the Phantom: Virtual touch through point interaction," Master thesis, MIT, 1996.

[10] C. Basdogan C. Ho and M. A. Srinivasan, "Efficient point-based rendering techniques for haptic display of virtual objects," Presence: Teleoperators and Virtual Environment, vol. 8, pp. 477-491, 1999.

[11] S. Choi and H.Z. Tan, "Perceived instability of virtual haptic texture. i. experimental studies," Presence: Teleoperators and Virtual Environments, vol. 13, pp. 395-415, 2004.

[12] J. J. Eggers and B. Girod, Informed Watermarking, Kluwer Academic Publishers, 2002.

[13] L. L. Scharf, Statistical Signal Processing: Detection, Estimation, and Time Series Analysis, AddisonWesley, Reading, MA, 1991.

[14] M. Barni and F. Bartolini, Watermarking Systems Engineering: Enabling Digital Assets Security and Other Applications, Marcel Dekker, 2004.
[15] S. J. Lederman, J. M. Loomis, and D. A. Williams, "The role of vibration in the tactual perception of roughness," Percept. Psychophys., vol. 32, pp. 109116,1982

[16] S. J. Lederman, "Tactual roughness perception: Spatial and temporal determinants.," Can. J. Psychol., vol. 37, pp. 498-511, 1983.

[17] C. E. Connor and K. O. Johnson, "Neural coding of tactile texture: Comparison of spatial and temporal mechanisms for roughness perception," J. Neurosci., vol. 12, pp. 3414-3426, 1992.

[18] J. M. Weisenberger and M. J. Krier, "Haptic perception of simulated surface textures via vibratory and force feedback displays," in Proc. of the ASME Dynamic Systems and Control Division, 1997, pp. 5560.

[19] A. M. Okamura, J. T. Dennerlein, and R.D. Howe, "Vibration feedback models for virtual environments," in Proceedings of the IEEE International Conference on Robotics and Automation, 1998, pp. 2485-2490.

[20] R. T. Verrillo, "Effect of contactor area on the vibrotactile threshold," J. Acous. Soc. Am., vol. 35, pp. 1962-1966, 1963.

[21] N. A. Macmillan and C. D. Creelman, Detection Theory: A User's Guide, Cambridge University Press, New York, USA, 1001.

[22] T.D. Wickens, Elementary Signal Detection Theory, Oxford University Press, New York, USA, 2002. 